\title{
A Prospective Study on the Efficacy and Safety of Kangaroo Mother Care as an Alternative Means of Transport of Preterm and Term Small-for-Gestational Age Infants
}

\author{
Gerlie Ann A. Zamora, MD and Daisy C. Garcia, MD \\ Ilocos Training and Regional Medical Center, San Fernando City, La Union, Philippines
}

\begin{abstract}
Background. The unavailability of transport incubators in resource-limited areas increases the risk for hypothermia in low birthweight neonates requiring transfer to another hospital. The kangaroo mother care (KMC) position may be a better alternative than swaddling the neonates during transport.

Objective. To determine the safety and efficacy of $\mathrm{KMC}$ as an alternative means of transport of preterm and term small-for-gestational age (SGA) infants who need to be transferred to a higher level of care. Specifically, it aims to establish if KMC is safe and efficacious in terms of thermoregulation during inter-facility transfers. It also aims to determine the impact of transport distance from the referring hospital, age of gestation, sex, birthweight, and Apgar score on the efficacy of KMC in preventing hypothermia.

Methods. We did a prospective, single-blinded, parallel-randomized controlled trial from September 2016 to October 2017 from a community-based primary care facility to a tertiary government hospital. We included newborn preterm infants and term SGA infants weighing 1200-1800 grams, delivered at health centers, district and provincial hospitals who needed to be transferred for a higher level of care. Outcomes included physiologic variables such as temperature, heart rate, respiratory rate. We conducted statistical analysis using t-test, risk ratio, and multiple regression analysis.
\end{abstract}

Results. Thirty-one neonates were randomized to KMC transport $(n=15)$ and conventional transport (swaddled) $(n=16)$. Fifty percent of the swaddled infants developed hypothermia against none in the KMC infants. The risk of hypothermia was reduced by $93.75 \%$ in the neonates transported in KMC. The gestational age, birthweight, sex, Apgar scores and distance travelled had no confounding effect on the neonates' temperature during transport.

Conclusions. Kangaroo mother care transport is a safe, effective, and low-cost alternative in inter-facility neonatal transport especially in limited-resource areas.

Key Words: kangaroo mother care, skin-to-skin contact, low birthweight, hypothermia, neonatal transport

\section{INTRODUCTION}

Corresponding author: Gerlie Ann A. Zamora, MD

llocos Training and Regional Medical Center

MacArthur Highway, San Fernando City 2500

La Union, Philippines

Email: geann1011@gmail.com
The first 28 days of life is the most vulnerable time for a child's survival. The average global rate is 17 deaths per 1,000 live births in 2019. Globally, 2.4 million children died during the first month and about third of which are occurring within the first day after birth. ${ }^{1}$ In the Philippines, an estimated 60000 of 1.7 million children born each year die under the age of five with around half occurring among newborns. ${ }^{2}$ 
A significant contributor to neonatal morbidity and mortality is hypothermia. In moderate hypothermia (between $32^{\circ} \mathrm{C}$ and $\left.36.4^{\circ} \mathrm{C}\right)$ and severe hypothermia $\left(<32^{\circ} \mathrm{C}\right)$, the baby would manifest with breathing difficulty, heart rate less than 100 beats per minute, poor or no feeding, and lethargy. Advancement in technology has brought incubators that can help improve outcomes in high-risk infants. ${ }^{3}$ However, such equipment is not readily available in low- to middleincome countries. With this kind of situation, interventions that are feasible and readily available in both high- and low-income settings are highly desired.

Kangaroo mother care (KMC) was introduced in Bogota, Columbia as an alternative to incubators for low birthweight (LBW) infants. KMC is a simple method of care for LBW infants that includes early and prolonged skinto-skin contact with the mother or a substitute caregiver. $\mathrm{KMC}$ compared to conventional care was associated with $36 \%$ lower mortality. KMC reduces the risk of neonatal sepsis, hypothermia, hypoglycemia, and hospital readmission and increased exclusive breastfeeding. ${ }^{4}$ It is one of the best evidence-based approach to reduce mortality and morbidity secondary to hypothermia. KMC was first established at Dr. Jose Fabella Memorial Hospital in Manila in 1999. Thereafter, the Department of Health (DOH) integrated and strengthened $\mathrm{KMC}$ through a national policy of care for preterm and LBW newborns. ${ }^{5}$

To date, there is no local policy enforcing the practice of $\mathrm{KMC}$ in inter-hospital referrals. Most neonatal transports are self-conduction without any pre-treatment stabilization or care during transport. Thus, many babies transported are received cold, blue and hypoglycemic. It is thus imperative to determine if neonatal transport in the $\mathrm{KMC}$ position during inter-facility transfers can lead to better thermoregulation of high-risk infants. Results from this study may lead to development of guidelines and policies on the conduct of neonatal transport in resource-limited areas.

\section{OBJECTIVES}

\section{General Objective}

To determine if neonatal transport in the $\mathrm{KMC}$ position during inter-facility transfers can lead to better thermoregulation of low birthweight infants

\section{Specific Objectives:}

1. To compare the temperature, heart rate, respiratory rate, and oxygen saturation among LBW infants on KMC transport versus local transport practices (swaddled) during inter-facility transfers

2. To determine the impact of transport distance from the referring hospital, age of gestation, sex, birthweight, and Apgar score on the efficacy of KMC in preventing hypothermia

\section{METHODS}

\section{Study Design}

We did a prospective, randomized, sequential clinical study from September 2016 to October 2017 among newborns transferred from primary care facilities to a tertiary government hospital, the Ilocos Training and Regional Medical Center (ITRMC). Randomization was achieved using computer-generated permuted blocks of 10 to receive either KMC or conventional method of transport (swaddled). The treatment allocation was concealed by placing the $\mathrm{KMC}$ and conventional kits in opaque, brown, and sealed envelopes. Because of the difference in appearance of the interventional kits, the caregivers who were responsible for the application of the kits and the guardians who served as kangaroos or companions of the babies were unmasked but were not included in the study analysis. The study investigator remained masked until the end of the study.

\section{Study Population}

We invited participants from birthing facilities in a community whose staff had undergone training course in $\mathrm{KMC}$. Only those centers who consented were included in the study.

Eligible participants included all hemodynamically stable neonates, with an Apgar score of $\geq 7$ at 1 and 5 minutes of life, and preterm or small-for-gestational age with a birth weight of 1200-1800 grams because these groups are the most vulnerable to changes in temperature. Infants needing ventilator support, intravenous fluids, vasopressors and had congenital anomalies were excluded from the study.

\section{Procedures}

To attain uniformity, each participating center was supplied with the same type of digital thermometer, pulse oximeter, and interventional kits. Both the conventional and $\mathrm{KMC}$ kits contained caps and blankets whereas KMC kit had an additional tube blouse, all made of the same materials. Caps and blankets were made of cotton while the $\mathrm{KMC}$ tube was made of spandex.

A written informed consent was obtained prior to the study and a copy of the signed consent was given to the parents. Participation was voluntary and withdrawal at any time during the study was respected. Confidentiality of participant data was also ensured.

In $\mathrm{KMC}$ transport (KT), the neonate was positioned following the WHO guidelines. ${ }^{6}$ The neonate was donned with a cap and diaper, transferred gently, and placed against the caregiver's bare chest, head was turned to one side and in a slightly extended position, arms were flexed, and legs were flexed and abducted in a "frog" position. After proper positioning of the neonate, he or she was secured using the KMC blouse worn by the caregiver. A blanket was placed over the infant. The caregiver was then instructed to place his/her hands to support the back and the neck. 
In conventional transport (CT) or usual transport practice (swaddled), the neonate was donned with a cap and cotton clothing and wrapped with a blanket. The neonate was then placed on a basinet/crib or handheld if not available.

Health workers were provided forms for instruction, data collection and monitoring. The heart rate, respiratory rate, body temperature, and oxygen saturation of participants were recorded by the health worker at the beginning of transport, during or midway of transport, and upon arrival at emergency room of ITRMC. The respiratory rate was counted for a full minute using a stethoscope. The heart rate and oxygen saturation were determined using the digital thermometer. The transport vehicle was an ambulance.

\section{Statistical Analysis}

Data were recorded on a predesigned template, tabulated and statistically analyzed. The baseline characteristics of participants were analyzed statistically by t-test to compare means of continuous variables. The mean chance on the physiologic responses in temperature of participants pretransport, during and upon arrival at ER were analyzed using the one tailed t-test. A p-value of $<0.05$ was considered significant.

The relative risk ratio was computed using OpenEpi. Regression analysis was used to determine the presence of confounding variables.

\section{RESULTS}

A total of 31 participants were recruited; 2 came from city health office, 18 came from district hospitals, and 11 from rural health units; the approximate distance from ITRMC ranged from 3-49 kilometers. The final study groups comprised of 15 neonates (10 boys, 5 girls) in the $\mathrm{KMC}$ transport group and 16 (7 boys, 9 girls) in conventional transport group.

Participants in the KMC transport group had a mean gestational age of 36 weeks, a mean weight of 1670 grams, and Apgar score of 7 (1 minute) and 9 (5 minutes of life). The conventional transport group participants had a mean gestational age of 35 weeks, mean weight of 1590 grams, and Apgar score of 8 (1 minute) and 9 (5 minutes of life). Statistically, there was no significant difference in the baseline characteristics between the groups except in their initial Apgar score (Table 1).

Hypothermia occurred in eight subjects in conventional transport group while none was noted in the KMC transport group (RR $0.063,95 \%$ CI of 0.004 to 0.997) (Table 2). The babies transported through kangaroo transport had a $93.75 \%$ reduction in the risk of hypothermia compared to conventional transport.

There was a notable difference in the physiologic status between the two groups particularly in their heart rate, respiration, and temperature (Table 3). The temperature of neonates in the kangaroo care transport group increased by $0.02^{\circ} \mathrm{C}$ and $0.48^{\circ} \mathrm{C}$ during and post-kangaroo transport. During KMC transport, most babies had a steady rise in temperature, and none developed hypothermia. The respiratory rate was decreased by 5 breaths per minute during $\mathrm{KMC}$ transport and heart rate was decreased by 5 beats per minute post-KMC transport. The heart and respiratory rate were modestly decreased in KMC transport; these changes were statistically significant.

Table 1. Baseline characteristics of participants

\begin{tabular}{|c|c|c|c|}
\hline \multirow[t]{2}{*}{ Characteristics } & $\begin{array}{c}\text { Kangaroo } \\
\text { transport }(n=15)\end{array}$ & $\begin{array}{c}\text { Conventional } \\
\text { transport }(n=16) \\
\end{array}$ & \multirow[t]{2}{*}{$P$} \\
\hline & \multicolumn{2}{|c|}{ Mean \pm SD } & \\
\hline Maternal age & $26.4 \pm 4.24$ & $25.63 \pm 5.69$ & 0.680 \\
\hline Age of gestation (weeks) & $36.07 \pm 1.39$ & $35.56 \pm 1.22$ & 0.307 \\
\hline Birth weight (grams) & $1670 \pm 0.17$ & $1590 \pm 0.17$ & 0.209 \\
\hline Apgar score (1 $\left.1^{\text {st }} \mathrm{min}\right)$ & $7.40 \pm 0.71$ & $8.13 \pm 0.99$ & $0.032^{*}$ \\
\hline Apgar score $\left(5^{\text {th }} \mathrm{min}\right)$ & $9.0 \pm 0.63$ & $8.9 \pm 0.30$ & 0.617 \\
\hline
\end{tabular}

${ }^{*}$ t-test significant if $p<0.05$

Table 2. Comparison of post-transport temperature between Kangaroo transport (KT) and Conventional transport (CT) on hypothermia

\begin{tabular}{|c|c|c|c|c|}
\hline \multirow{2}{*}{ Intervention } & \multicolumn{2}{|c|}{ Temperature post transport } & \multicolumn{2}{|c|}{ Results* } \\
\hline & $<36.5^{\circ} \mathrm{C}$ & $36.5-37.5^{\circ} \mathrm{C}$ & Risk Ratio & $95 \% \mathrm{Cl}$ \\
\hline$K T(n=15)$ & 0 & 15 & \multirow{2}{*}{0.0625} & $\begin{array}{c}0.00392 \\
0.9965\end{array}$ \\
\hline$C T(n=16)$ & 8 & 8 & & \\
\hline
\end{tabular}

*where zeros cause problems with computation of the relative risk or its standard error, 0.5 is added to all cells $(a, b, c, d)$ (Pagano \& Gauvreau, 2000; Deeks \& Higgins, 2010)

Table 3. Comparison of physiologic variables between Kangaroo transport (KT) and Conventional transport (CT)

\begin{tabular}{|c|c|c|c|}
\hline \multirow{2}{*}{$\begin{array}{l}\text { Characteristics } \\
\text { (Transport Period) }\end{array}$} & $\mathrm{KT}(\mathrm{n}=15)$ & CT $(n=16)$ & \multirow{2}{*}{$P$} \\
\hline & \multicolumn{2}{|c|}{ Mean \pm SD } & \\
\hline \multicolumn{4}{|l|}{ Temperature $\left({ }^{\circ} \mathrm{C}\right)$} \\
\hline Prior & $36.52 \pm 0.32$ & $36.48 \pm 0.24$ & 0.66 \\
\hline During & $36.69 \pm 0.25$ & $36.47 \pm 0.28$ & $0.02^{*}$ \\
\hline After & $36.85 \pm 0.06$ & $36.37 \pm 7.38$ & $<0.001^{*}$ \\
\hline \multicolumn{4}{|l|}{ Heart rate (bpm) } \\
\hline Prior & $143.75 \pm 6.85$ & $148.60 \pm 8.20$ & 0.09 \\
\hline During & $144.63 \pm 6.14$ & $149.13 \pm 7.87$ & 0.09 \\
\hline After & $146.00 \pm 4.20$ & $151.07 \pm 7.38$ & $0.02^{*}$ \\
\hline \multicolumn{4}{|l|}{ Respiration (bpm) } \\
\hline Prior & $53.19 \pm 7.72$ & $59.20 \pm 5.84$ & $0.02^{*}$ \\
\hline During & $50.38 \pm 6.13$ & $59.27 \pm 5.12$ & $0.00^{*}$ \\
\hline After & $49.6 \pm 5.26$ & $48.13 \pm 4.03$ & 0.59 \\
\hline \multicolumn{4}{|l|}{$\mathrm{O}_{2}$ saturation (\%) } \\
\hline Prior & $93.93 \pm 1.91$ & $94.06 \pm 1.73$ & 0.84 \\
\hline During & $95.47 \pm 1.26$ & $96.06 \pm 2.06$ & 0.35 \\
\hline After & $97.27 \pm 1.24$ & $96.56 \pm 1.44$ & 0.57 \\
\hline
\end{tabular}

${ }^{*}$ One-tailed t-test significant if $p<0.05$

bpm - beats per minute 
Table 4. Effect of distance, sex, birth weight, Apgar score to temperature in Kangaroo transport

\begin{tabular}{lcccccc}
\hline & Coefficients & Standard Error & t stat & P-value & Lower 95\% & Upper 95\% \\
\hline Intercept & 37.18 & 1.66 & 22.46 & 0.00 & 33.75 & 40.60 \\
Treatment & 0.46 & 0.11 & 4.15 & 0.00 & 0.23 & 0.69 \\
Distance from ITRMC & 0.01 & 0.00 & 2.03 & 0.05 & 0.00 & 0.02 \\
Pediatric aging & -0.03 & 0.04 & -0.78 & 0.44 & -0.12 & 0.05 \\
Sex & -0.01 & 0.11 & -0.08 & 0.94 & -0.24 & 0.22 \\
Birth weight & 0.04 & 0.31 & 0.12 & 0.91 & -0.61 & 0.68 \\
Apgar score at 1 $\mathrm{min}$ & 0.03 & 0.09 & 0.32 & 0.75 & -0.16 & 0.22 \\
Apgar score at 5 $\mathrm{min}$ & -0.06 & 0.11 & -0.55 & 0.59 & -0.28 & 0.16
\end{tabular}

Only the exposure to kangaroo transport had significant correlation with the temperature (Table 4). There was a significant increase in temperature regardless of factors such as distance of travel and newborn characteristics (pediatric aging, sex and weight). Although Apgar score was initially noted to have a significant difference (Table 1 ), the regression analysis demonstrated that Apgar score did not show significant correlation with temperature.

\section{DISCUSSION}

This study demonstrated that KMC transport could significantly prevent the development of hypothermia among infants undergoing inter-facility transfers. KMC is a simple, low-cost intervention for the care of LBW infants and can be practiced in any situation without needing special equipment. Hypothermia has significant impact on neonatal morbidity and mortality. During KMC transport, the infant experiences maternal heart sounds, rhythmic maternal breathing, warmth, and prone positioning, all of which offer gentle stimulation across auditory, tactile, vestibular, and thermal sensory systems, which may in total have a tranquilizing effect on the baby, allowing physiological parameters to stabilize. ${ }^{7}$

The rapid heat loss occurs in preterm infant because of larger surface area to body mass ratio, weak muscle tone/ poor flexion, thinner immature skin, increased evaporative water loss, poor ability to vasoconstrict, decreased amounts of insulating fat and reduced amount of brown fat. ${ }^{8}$ Heat is lost by conduction, convection, radiation, and evaporation. ${ }^{8}$ As body temperature falls outside of the normal range of $36.5^{\circ} \mathrm{C}$ to $37.5^{\circ} \mathrm{C}$, metabolic rate increases to produce and conserve heat, which in turn increases oxygen consumption and glucose utilization. This is more pronounced in infants requiring resuscitation and if immediate intervention is not done, metabolism ensues leading to lactic acidosis and eventually death. Although this highly preventable condition has long been recognized, it continues to be a problem in neonatal care. Therefore, assisting the infant to maintain a normal body temperature and preventing the consequences of hypothermia is critically important.
Following birth, interventions to decrease the risk of hypothermia include thorough drying, immediate skin to skin contact, removing wet linens, use of warm blankets and cap and maintaining the mother and the baby dyad even after the initial breastfeeding. ${ }^{9}$ The use of plastic and aluminum foil wrap, radiant warmer and incubator are used if the baby remains hypothermic. However, these modern technologies were not available, skin-to-skin contact is a lowcost alternative. In a study by Bergman, skin-to-skin care was shown to be effective in reducing the risk of hypothermia when compared to conventional incubator care for infants. ${ }^{10}$

Previous studies on thermoregulation and $\mathrm{KMC}$ involved LBW infants admitted in the NICU. A randomized controlled trial done on 242 stable LBW babies weighing less than 2000 grams showed that the incidence of hypothermia was also lower in the KMC group (5.1\%) compared with the control group (14.6\%). ${ }^{11} \mathrm{~A}$ meta-analysis of 124 preterm and LBW infants consisting of observational and randomized trials comparing $\mathrm{KMC}$ and conventional care also showed a significantly lower incidence of hypothermia among infants on $\mathrm{KMC}(\mathrm{RR} 0.22,95 \% \mathrm{CI} 0.12,0.41) .{ }^{12}$

There is a paucity of published data on neonatal transport using the KMC position. In a 2004 descriptive study by Sontheimer et al., 31 stable preterm and term infants who required transfers and back transfers to the hospital (2-to400-kilometer distance) using KMC transport were observed to remain physiologically stable (heart rate, respiratory rate, oxygen saturation, and rectal temperature).${ }^{13}$ In our study, the distances travelled were still relatively far at 3 to $49 \mathrm{~km}$. But unlike the study by Sontheimer et al., where the transport was through land (ambulances) and air (helicopters), the inter-facility transfers in our study were all land travels using public utility vehicles (Philippine jeepney) and tricycles (motorcycle with side car). Furthermore, environmental temperature can be controlled in the ambulances and helicopters. This was not possible in our study since the infants were transported in vehicles without any airconditioning units and may even have had open side panels (e.g., tricycles and jeepneys). As such, the risk for hypothermia is truly considerable in resource-limited areas, not only due to the absence of a transport incubator, but also due to uncontrolled ambient temperatures in the vehicles used. 
The first randomized controlled trial on KMC transport was done by Ignacio ${ }^{14}$ at the Philippine General Hospital. In this trial, the KMC transport was compared with the gold standard of neonatal transport, the transport incubator. The study demonstrated that the physiologic responses (including temperature, heart rate, respiratory rate, oxygen saturation and capillary blood glucose) did not significantly differ between $\mathrm{KMC}$ transport and transport by incubator. This emphasized that KMC transport is comparable in effectiveness with the transport incubator, the gold standard, on thermoregulation and other physiologic parameters. In our study, the comparator group was not the standard of care, but rather the usual care in resource-limited settings. There were neither transport incubators nor ambulances readily available for use. KMC transport significantly prevented hypothermia (97\%) compared with the usual practice of infant swaddling, which resulted in 50\% of the infant's developing hypothermia. This study revealed that $\mathrm{KMC}$ transport is far more superior than our usual transport practice.

The study by Ignacio dealt with in-hospital transfers from delivery room to NICU with the infant in KMC position and the caregiver seated in a wheelchair. Our study is the first randomized controlled trial on inter-facility neonatal transfers using KMC transport in resource-limited areas.

The strength of our study is in its application of KMC practice in neonatal transport. The geographic inaccessibility of birthing centers to land transportation and the distance to referral centers make neonatal transport difficult. Our experience has shown that most of the babies referred for further care are admitted hypothermic. Transport using $\mathrm{KMC}$ can significantly reduce the incidence of hypothermia because thermoregulation can be done immediately and continuously while on transport without use of special equipment, thus improving the outcome of the babies.

\section{CONCLUSION}

KMC transport is protective against hypothermia and has not shown evidence of harm. This safe and lowcost intervention has the potential to prevent many complications associated with preterm birth and LBW in full term newborns. Stronger local evidence on KMC transport should be obtained through larger-sized studies. This will inform clinical guidelines and policy development, including widespread implementation as a standard of care for newborns.

\section{Statement of Authorship}

Both authors contributed equally in the conceptualization of work, acquisition and analysis of data, drafting and revising and approved the final version submitted.

\section{Author Disclosure}

Both authors declared no conflicts of interest.

\section{Funding Source}

The study has no funding support.

\section{REFERENCES}

1. UNICEF. Neonatal mortality/Child mortality data [Internet]. 2020 [cited 2021 Oct]. Available from https://data.uniceforg/topic/childsurvival/neonatal-mortality/

2. Philippines Statistics Authority. Vital Statistics 2018 - Preliminary Counts of Births, Marriages and Deaths. Republic of the Philippines [Internet]. 2019 [cited 2021 Oct]. Available from https://psa.gov. $\mathrm{ph} /$ civilregistration/vital-statistics-2018-preliminary-counts-birthsmarriages-and-deaths

3. World Health Organization. Managing Newborn Problems. Hong Kong, WHO publications [Internet]. 2013 [cited 2018 Mar]. Available from https://apps.who.int/iris/bitstream/handle/ 10665/42753/9241546220.pdf

4. Joint statement International Policy Statement for universal use of Kangaroo Mother Care for preterm and low birthweight infants [Internet]. 2016 [cited 2018 Mar]. Available from http://www. healthynewbornnetwork.org/resource/KMCjointstement

5. Calibo AP, De Leon Mendoza S, Silvestre MA, Scott Murray JC, Li Z, Mannava P, et al. Scaling up kangaroo mother care in the Philippines using policy, regulatory and systems reform to drive changes in birth practices. BMJ Global Health. 2021 Aug; 6(8). doi: 10.1136/bmjgh-2021-006492. PMID: 34417273; PMCID: PMC8381328.

6. Department of Reproductive Health and Research Work. Kangaroo mother care: A practical guide. Geneva: World Health Organization [Internet]. 2003 [cited $2018 \mathrm{Mar}$ ]. Available from: http://apps.who. int/iris/bitstream/handle/10665/42587/9241590351.pdf;jsessionid=3AB7909D4A90991083D7F58F5350E95B? sequence $=1$

7. Cloherty J, Eichenwald E, Hansen A, Stark A. Temperature control. In: Chatson KE. Manual of Care, 7th ed., Philadelphia, PA, Lippincott Williams \& Wilkins, 2012. pp. 178-184

8. Kalsen K. The S.T.A.B.L.E. Program Pre-transport/ Post resuscitation stabilization care of sick infants' guidelines for neonatal healthcare providers, 6th ed. Salt Lake City, S.T.A.B.L.E. Inc., 2013. pp. 69-74

9. McCall EM, Alderdice FA, Halliday HL, Jenkins JG, Vohra S. Interventions to prevent hypothermia at birth in preterm and/or low birthweight babies. Cochrane Database Syst Rev. 2005 Jan 25; (1):CD004210. doi: 10.1002/14651858.CD004210.pub2. Update in: Cochrane Database Syst Rev. 2008;(1):CD004210. PMID: 15674932.

10. Bergman NJ, Linley LL, Fawcus SR. Randomized controlled trial of skin-to-skin contact from birth versus conventional incubator for physiological stabilization in 1200 - to 2199 -gram newborns. Acta Paediatr. 2004 Jun; 93(6):779-85. doi: 10.1111/j.1651-2227.2004. tb03018.x. PMID: 15244227.

11. Kanodia P, Bora R, Gupta A. Kangaroo mother care- a cost effective and an alternate method to manage hypothermia in low birthweight babies for better clinical outcome. Value Health. 2016 Nov; 19(7): A405. doi: https://doi.org/10.1016/j.jval.2016.09.338.

12. Boundy EO, Dastjerdi R, Spiegelman D, Fawzi WW, Missmer SA, Lieberman E, et al. Kangaroo mother care and neonatal outcomes: a meta-analysis. Pediatrics. 2016 Jan; 137(1):e20152238. doi: 10.1542/ peds.2015-2238. PMID: 26702029; PMCID: PMC4702019.

13. Sontheimer D, Fischer CB, Buch KE. Kangaroo transport instead of incubator transport. Pediatrics. 2004 Apr; 113(4):920-3. doi: 10.1542/peds.113.4.920. PMID: 15060247.

14. Ignacio R, Uy ME, Mantaring JB. Kangaroo care transport versus transport incubator in transporting stable preterm neonates: a randomized controlled trial. (Unpublished). 